\section{In Reply: More on Burnout and Surgical Oncology}

Dr. Hena raises interesting questions regarding (1) how burnout changes as surgical oncologists move from fellowship to practice, (2) when burnout begins, and (3) whether individuals at risk could be identified prior to residency/fellowship. With respect to the first question, although the total number of hours worked per week and the frequency of overnight call often decline after completing fellowship, this is not universally true. In our study of the Society of Surgical Oncology, 24\% of surgical oncologists worked more than $70 \mathrm{~h}$ per week and $12 \%$ were on call 5 or more nights per week. ${ }^{1}$ In addition, surgical oncologists entering practice face a number of new challenges such as managing an office, greater exposure to reimbursement issues, higher productivity expectations, and a need to establish their reputation within their practice and community. It is difficult to know how trading one set of stressors for a different set influences the experience of burnout, and it likely varies for each individual. Similarly, although as a generalization, surgeons earlier in the training process (medical school and residency) may experience less distress related to tension between personal and professional responsibilities, this again varies for each individual. Indeed, a sizable proportion of medical students and residents are married and have children, which may force them to deal with stress related to balance between personal and professional life earlier in their career than those surgeons who do not get married or have children until after they are in practice.

At the cohort level, it appears that the prevalence of burnout is lowest at the start of medical school, increases toward the end of medical school training, peaks during residency, and then subsequently declines after physicians enter practice. $^{2,3}$ Such population-level data, however, ignores the more nuanced experience of the individual surgeon where the first experience of burnout may not occur in a given individual until mid career. Indeed, our own longitudinal studies of burnout suggest that burnout varies through time and that the adverse consequences associated with burnout may be reversible. ${ }^{4}$ In this respect, it is important for surgeons to take inventory with respect to which aspects of their professional life are most or least satisfying and attempt to tailor their practice in such a way as to maximize professional satisfaction. Since preferences change through the course of a career, it cannot be assumed that the activities that were most satisfying when a surgeon first entered practice will necessarily be the factors that continue to provide meaning 10 years later. ${ }^{5}$ Developing the habit of periodic self-appraisal of personal and professional preferences early in a career may help surgeons make incremental adjustments that allow them to continually tailor their career to achieve greater satisfaction.

Finally, although some have advocated identifying individuals at greatest risk for burnout prior to acceptance into medical school or residency training in the hopes of "weeding out those at risk," this may be a dangerous approach. It is believed that those individuals who are most committed to and passionate about their work may be at greatest risk for experiencing work overload and/or burnout. Accordingly, selecting out those "at risk" may have the unintended consequence of removing some of the most talented and committed future surgeons from the field. Instead, we would advocate developing strategies that help surgeons promote personal well-being, identify early signs of burnout, and employ strategies to prevent it.

\section{Tait Shanafelt, M.D.}

Mayo Clinic, Rochester, MN, USA

e-mail: Shanafelt.Tait@mayo.edu

Published Online: 13 November 2008

(C) Society of Surgical Oncology 2008

\section{REFERENCES}

1. Kuerer HM, Eberlein TJ, Pollock RE, et al. Career satisfaction, practice patterns and burnout among surgical oncologists: report on the quality of life of members of the Society of Surgical Oncology. Ann Surg Oncol. 2007;14:3043-53.

2. Dyrbye LN, Thomas MR, Shanafelt TD. Medical student distress: causes, consequences, and proposed solutions. Mayo Clin Proc. 2005;80:1613-22.

3. Dyrbye LN, Thomas MR, Huntington JL, et al. Personal life events and medical student burnout: a multicenter study. Acad Med. 2006;81:374-84.

4. Dyrbye LN, Thomas MR, Massie FS, et al. Burnout and suicidal ideation among U.S. medical students. Ann Intern Med. 2008;149: 334-41.

5. Shanafelt T, Chung H, White H, Lyckholm LJ. Shaping your career to maximize personal satisfaction in the practice of oncology. J Clin Oncol. 2006;24:4020-6. 